\title{
Radio-Frequency Inductor Synthesis Using Evolutionary Computation and Gaussian-Process Surrogate Modeling
}

\author{
F. Passos, E. Roca, R. Castro-López, F.V. Fernández \\ Instituto de Microelectrónica de Sevilla, IMSE-CNM, CSIC and Universidad de Sevilla. \\ Calle Américo Vespucio, s/n, Isla de la Cartuja, 41092 Sevilla, Spain. \\ Corresponding author: Fábio Passos (moreira@imse-cnm.csic.es)
}

\begin{abstract}
In recent years, the application of evolutionary computation techniques to electronic circuit design problems, ranging from digital to analog and radiofrequency circuits, has received increasing attention. The level of maturity runs inversely to the complexity of the design task, less complex in digital circuits, higher in analog ones and still higher in radiofrequency circuits. Radiofrequency inductors are key culprits of such complexity. Their key performance parameters are inductance and quality factors, both a function of the frequency. The inductor optimization requires knowledge of such parameters at a few representative frequencies. Most common approaches for optimization-based radiofrequency circuit design use analytical models for the inductors. Although a lot of effort has been devoted to improve the accuracy of such analytical models, errors in inductance and quality factor in the range of $5 \%$ to $25 \%$ are usual and it may go as high as $\mathbf{2 0 0 \%}$ for some device sizes. When the analytical models are used in optimization-based circuit design approaches, these errors lead to suboptimal results, or, worse, to a disastrous non-fulfilment of specifications. Expert inductor designers rely on iterative evaluations with electromagnetic simulators, which, properly configured, are able to yield a highly accurate performance evaluation. Unfortunately, electromagnetic simulations typically take from some tens of seconds to a few hours, hampering their coupling to evolutionary computation algorithms. Therefore, analytical models and electromagnetic simulation represent extreme cases of the accuracy-efficiency trade-off in performance evaluation of radiofrequency inductors. Surrogate modeling strategies arise as promising candidates to improve such trade-off. However, obtaining the necessary accuracy is not that easy as inductance and quality factor at some representative frequencies must be obtained and both performances change abruptly around the self-resonance frequency, which is particular to each device and may be located above or below the frequencies of interest. Both, offline and online training methods will be considered in this work and a new two-step strategy for inductor modeling is proposed that significantly improves the accuracy of offline methods. The new strategy is demonstrated and compared for both, singleobjective and multi-objective optimization scenarios. Numerous experimental results show that the proposed two-step approach outperforms simpler application strategies of surrogate modelling techniques, getting comparable performances to approaches based on electromagnetic simulation but with orders of magnitude less computational effort.
\end{abstract}

Keywords

Surrogate models, evolutionary algorithms, single-objective optimization, multi-objective optimization, integrated inductors.

\section{Introduction}

Due to the complexity of analog and radio-frequency (RF) integrated circuit design and the demand for short time-to-market, there is a need for robust and efficient automated design methods. The design of an RF subsystem is usually performed by decomposing it into several sub-blocks, which are further decomposed down to the device level. This hierarchical decomposition can be traversed bottom-up or top-down [1]. In top-down hierarchical synthesis, the top level is designed first, resulting in the specifications for the sub-blocks. This process continues down to the lowest level. By the contrary, bottom-up synthesis methods designs the lowest level blocks first, and, then, compose the information of lower level sub-blocks up the hierarchy. Evolutionary computation algorithms may sustain both design methodologies; either through single-objective optimizations (e.g. top-down methods) or multi-objective optimization (e.g. bottom-up methods) [1].

In this paper we will focus on one of the most challenging tasks in RF integrated circuit design: the design of passive devices, e.g., integrated inductors, which are essential components of RF integrated circuits [2]. While accurate analytical models are available for active devices to be used in circuit simulators like SpectreRF [3] or HSPICE RF [4], this is not the case for inductors. Typically, designers rely on electromagnetic (EM) simulators, which are the most accurate performance evaluators for these devices. However, EM simulations are computationally intensive. With nowadays' demand for short time-to-market, the usage of iterative EM simulations during the design process is becoming unaffordable; therefore other alternatives must be explored.

In order to reduce the computational effort in the RF design process, designers have developed physical/analytical equivalent models [5]-[7]. However, these models fail to accurately model the complete useful region of the inductor design space. 
In the past few years, surrogate models have been used to replace complex computationally expensive simulation processes by simpler models that can be much more efficiently evaluated [8]. However, these surrogate models still show relatively high errors when trying to model the entire design space.

When these three alternatives for performance evaluation are embedded into an iterative optimization loop for inductor synthesis, different trade-offs between accuracy and efficiency arise. When EM simulation is used, the highest accuracy is achieved at the price of the highest computation time, usually in the range of days. Despite this limitation, they have shown their potentialities especially with multi-objective optimization techniques due to their specification-independent use [9]. Optimization techniques with physical/analytical models for performance evaluation lie at the opposite end of the accuracy-efficiency tradeoff: short synthesis times, typically in the range of minutes can be achieved, but with the lowest accuracy, because, generally, equivalent circuit models do not allow an accurate modeling of RF passive components [10].

Such efficiency-accuracy trade-off is significantly improved by the use of surrogate models [11]. However, the difficulties in generating surrogate models that allow an accurate modeling of the entire design space have hampered the development of these approaches. In order to overcome the inaccuracy problem of the previous synthesis strategies, new approaches are being developed based on an initial coarse model, which is locally improved during the optimization process, and have been successfully applied to diverse device and circuit synthesis problems [12]-[14]. Assuming that the convergence to the global optimum is achieved, the price to pay is higher computational time, as expensive EM simulations must be executed during the optimization process.

In this paper, the different synthesis techniques are discussed and compared for single- and multi-objective optimization of integrated spiral inductors. Then, an intelligent global surrogate modeling methodology is proposed, that is able to predict highly accurate inductor performances over the entire design space and provide comparable results to EM-based synthesis techniques in optimization problems, while enabling the reduction of the synthesis time by several orders of magnitude. In this way, the efficiency-accuracy trade-off for the real world application under exam can be pushed well ahead available methods.

The paper is organized as follows. In Section 2, the inductor synthesis problem is formulated as an optimization problem and the algorithms used in this paper are briefly described. Section 3 overviews the different synthesis techniques reported in the literature, while Section 4 describes the proposed surrogate modeling methodology. This methodology is then used in Section 5 in several single-objective optimization problems, and results for the different surrogate-based synthesis techniques presented in Section 3 are compared. Afterwards, the new surrogate modeling methodology is used for multi-objective inductor optimization and comparisons are made against the same optimizations based on EM simulations. Finally, conclusions are drawn out in Section 6 .

\section{Problem Formulation}

Inductors in RF integrated circuits are typically built by using two metal layers (usually the upper ones, to minimize substrate losses) with an intermediate dielectric layer. As illustration's example, Fig. 1 shows the shape of an octagonal asymmetric spiral inductor. The geometry of this planar spiral inductor is usually defined by four geometric parameters: number of turns $(N)$, inner diameter $\left(D_{\text {in }}\right)$, turn width $(w)$ and spacing between turns $(s)$.

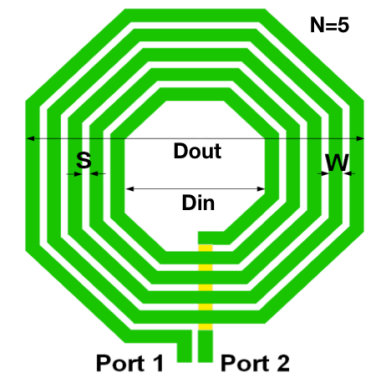

Fig. 1. Inductor geometric parameters for an octagonal asymmetric spiral inductor.

The most relevant inductor performances are the equivalent inductance, $L_{e q}$, and the quality factor, $Q$, which are defined as:

$$
\begin{aligned}
L_{e q}(f) & =\frac{\operatorname{Im}\left[Z_{e q}(f)\right]}{2 \pi f} \\
Q(f) & =\frac{\operatorname{Im}\left[Z_{e q}(f)\right]}{\operatorname{Re}\left[Z_{e q}(f)\right]}
\end{aligned}
$$

where $f$ is the frequency and $Z_{e q}$ is the equivalent input impedance. The equivalent input impedance can be easily obtained 
from the scattering parameters of the two-port structure representation of the inductor [15].

In Fig. 2, three different plots of the inductance and quality factor as a function of the frequency are illustrated. An important parameter is the self-resonance frequency, $S R F$, which is defined as the frequency at which the imaginary part of $Z_{e q}$ is zero, or, equivalently, the frequency at which the behavior of the inductor changes from inductive to capacitive (see (1)).
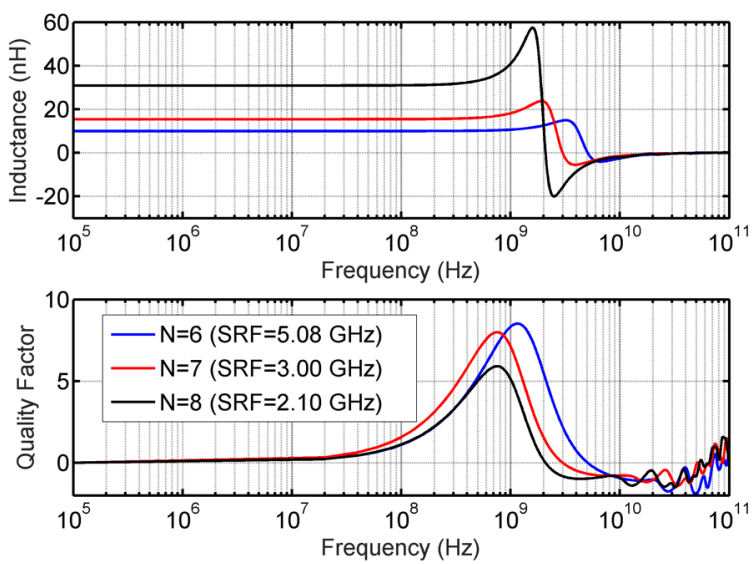

Fig. 2. Illustrating inductance and quality factor as a function of frequency for three different inductors.

The designer is usually interested in obtaining a given inductance at the frequency of operation, with the largest quality factor, and with the smallest area occupation since fabrication cost grows linearly with area, and inductors represent a large percentage of the RF circuit area. These three parameters: inductance, quality factor and area, are mutually conflicting. In addition, to minimize performance degradations due to small variations, the inductance should be sufficiently flat from low frequencies up to around the frequency of operation and the self-resonance frequency should be sufficiently above the frequency of operation [9]. Consequently, the inductor optimization problem can be posed as a constrained optimization problem:

$$
\begin{array}{cl}
\text { maximize } \boldsymbol{F}(\boldsymbol{x}) ; & \boldsymbol{F}(\boldsymbol{x})=\left\{f_{1}(\boldsymbol{x}), . ., f_{n}(\boldsymbol{x})\right\} \in \mathbb{R}^{n} \\
\text { such that: } \boldsymbol{G}(\boldsymbol{x}) \geqslant 0 ; & \boldsymbol{G}(\boldsymbol{x})=\left\{g_{1}(\boldsymbol{x}), . ., g_{m}(\boldsymbol{x})\right\} \in \mathbb{R}^{m} \\
& \text { where } x_{L i} \leqslant x_{i} \leqslant x_{U i}, i \in[1, p]
\end{array}
$$

where $\boldsymbol{x}$ is a vector with $p$ geometric parameters, each design parameter being restricted between a lower limit $\left(x_{L i}\right)$ and an upper limit $\left(x_{U i}\right)$. The functions $f_{j}(\boldsymbol{x})$, with $1 \leq j \leq n$, are the objectives that will be optimized, where $n$ is the total number of objectives. The functions $g_{k}(\boldsymbol{x})$, with $1 \leq k \leq m$, are design constraints which can be defined independently for each optimization problem.

If $n=1$ then the optimization problem is single-objective, and, if $n>1$, it is multi-objective. Whereas the solution to the former is a single design point, the solution to the latter is a set of solutions, the Pareto set, exhibiting the best trade-offs between the objectives, i.e., the Pareto optimal front (PF).

In this work, the Particle Swarm Optimization (PSO) algorithm [16] is used for single-objective optimization. As in evolutionary algorithms, the system is initialized with a population of random solutions and searches for optimal solutions along a set of iterations. In PSO, solutions are represented by particles that have a position and a velocity (providing the position of the particle at the following iteration). Each particle velocity is updated with a combined influence of its own inertia, its historical best position and the position of the best neighboring particle.

The population-based evolutionary optimization algorithm NSGA-II [17] was selected as multi-objective optimization algorithm in our approach. NSGA-II is based on the concept of Pareto dominance and ranking of solutions, and the output of the algorithm is a non-dominated set of points in the feasible objective space, i.e., the Pareto-optimal front.

The inductor design problem is a constrained optimization problem, as shown in (3). NSGA-II was designed to handle constrained multi-objective optimization problems. However, the standard PSO algorithm was designed to only deal with unconstrained optimization problems. Therefore, a tournament selection method [18] has been implemented in PSO to handle design constraints: 


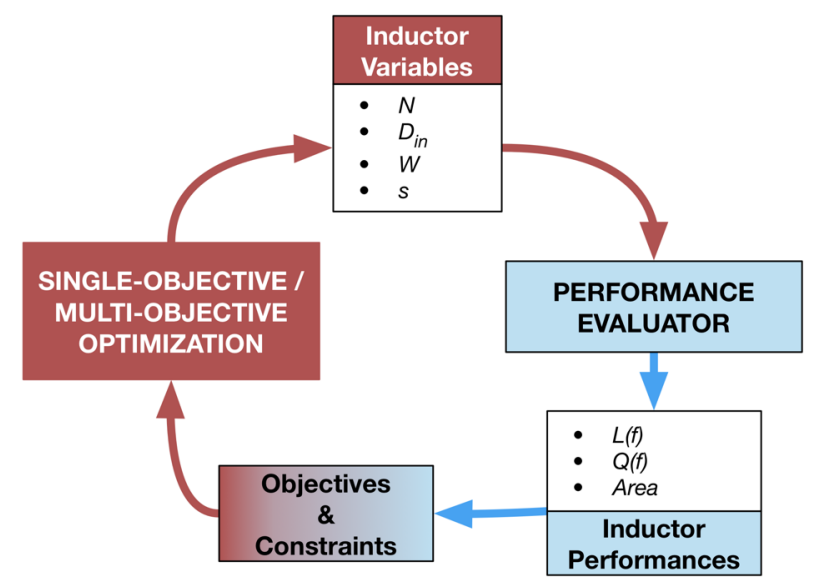

Fig. 3. Optimization-based inductor synthesis loop.

1) If two infeasible solutions are compared, the one with the smallest constraint violation is selected.

2) If one solution is feasible and another one is infeasible, the feasible one is selected.

3) If two feasible solutions are compared, the one with the best objective function is selected.

The work reported here does not exploit any specific characteristics of PSO and NSGA-II, hence, they can be replaced by any other single-objective and multi-objective optimization algorithm, respectively.

\section{Synthesis techniques}

The basic optimization-based synthesis flow for integrated inductors is illustrated in Fig. 3. The synthesis strategies can be classified in four categories according to the kind of performance evaluator and how it interacts with the optimization technique. They will now be discussed in detail, highlighting their advantages and drawbacks.

1) EM simulation as a performance evaluator of an optimization technique (EMO):

The EMO methods embed EM simulations to evaluate objectives and constraints within an optimization algorithm. Therefore, they provide the most accurate performance evaluation and, hence, the best solution quality of all methods. Their major drawback is the high computational cost of the EM simulations. Despite this limitation, they have shown their potentialities in multi-objective optimization-based bottom-up synthesis methodologies [9],[19]. Besides, it constitutes an excellent comparison benchmark for other techniques.

2) Equivalent circuit model as a performance evaluator of an optimization technique (ECO):

The ECO methods rely on a physical/analytical equivalent circuit model to obtain the performances of the passive component. Their main advantage is their high efficiency. However, reported equivalent circuit models are usually not accurate enough for a passive component synthesis, showing errors typically much higher than $10 \%$ [10]. Hence, when coupled with optimization algorithms, large deviations are observed on the synthesized passive components when their performances are verified with EM simulation. Therefore, these models can only be used for a first order approximation and not for a full inductor synthesis.

3) Offline surrogate model as a performance evaluator of an optimization technique (OFFSO):

With the OFFSO methodology, a global surrogate model is created before being used within an optimization algorithm [11]. The surrogate model is first built to be as accurate as possible. Then, the optimization algorithm uses this surrogate model as the performance evaluator to find the optimal solution. The surrogate model is called offline because training data can be generated by EM simulation and the model can be constructed before any optimization objectives and/or constraints are set.

Normally, the data set used to build the surrogate model is generated covering the entire design space. When combined with an optimization algorithm, this method has the ability of searching through the entire design space in order to find a global optimum. The generation of the training data is computationally expensive. However, such training data is generated only once and is valid for any future optimization problem. Moreover, they can be generated offline, much before they are needed for the first inductor optimization problem. On the other hand, since the model can be evaluated very fast, the optimization process itself is highly efficient, usually in the range of few minutes.

4) Online surrogate model as a performance evaluator of an optimization technique (ONSO):

Since global surrogate models may be locally inaccurate, ONSO methodologies, also known as surrogate-assisted evolutionary algorithms, have received considerable attention, also in practical analog/RF circuit design problems [14]. In this methodology, a 
coarse surrogate model using a few training points is first constructed. Then, this coarse model is coupled with an optimization algorithm and promising solutions (typically one) are electromagnetically simulated at each iteration of the optimization loop. The data from this EM simulation is used to update the surrogate model to make it more accurate in the region where new simulation points are added, while moving towards the presumed optimal inductor. However, the outputs of the ONSO methodology highly depend on the accuracy of the initial coarse model, which leads to two significant challenges for this methodology. First, the promising solutions found at the different iterations define the search space and the constructed surrogate model is only accurate in that space. Second, the success of ONSO comes from the basic assumption that the optimal point of the coarse and fine models are not far away in the design space. However, again, this assumption only holds when the coarse model is accurate enough.

ONSO methods exhibit a delicate trade-off between efficiency and the probability of convergence to the global optimum. Better convergence can be achieved by either increasing the amount of training data of the coarse model, or emphasizing the exploration of potentially good regions of the design space during the optimization process, or a combination of both. In all cases, an increase in the number of EM simulations is implied, diluting in this way the efficiency advantage over EMO methods.

A prescreening technique that can be used in ONSO methods in order to increase accuracy consists in using the uncertainty measurement of the prediction, i.e., the mean square error, instead of just the predicted value to rank promising solutions. Such techniques include methods like lower confidence bound, probability of improvement or expected improvement (EI). These methods have been widely applied for single-objective optimization [20],[21] and some more recent attempts have tried to extend these approaches to the multi-objective case [22]-[24]. Some of these approaches, especially the single-objective ones, have found their path to electronic circuit design application [14].

In this work, these approaches are examined and compared from the point of view of a real world application. In the surrogate model-based optimization of RF inductors proposed in this paper, efficiency is pushed forward by reclaiming the philosophy of OFFSO methods, and the improvement of accuracy is pursued by a new surrogate modeling strategy.

\section{Surrogate modeling methodology}

Surrogate modeling is an engineering method used when an outcome of interest of a complex system cannot be easily (or cheaply) measured either by experiments or simulations. An approximate model of the outcome is used instead. This section first describes the basic steps involved in the generation of a surrogate model, and afterwards presents the proposed methodology for modeling of the inductor performances.

\subsection{Surrogate modelling steps}

Generating a surrogate model usually involves four steps which are briefly described below, indicating the options adopted in this work.

\section{1) Design of experiments:}

The objective of surrogate models is to emulate the output response of a given system. Therefore, the model has to learn how the system responds to a given input. So, the first step in generating surrogate models is to select the input samples from which the model is going to learn. These samples should evenly cover the design space, so that it can be accurately modeled. In order to perform this sampling, different techniques are available, from classical Monte Carlo to Quasi-Monte Carlo or Latin Hypercube Sampling (LHS) [8]. In this work, LHS is used.

2) Accurate expensive evaluation:

Surrogate models learn from accurate but expensive evaluations. In our work, these accurate evaluations are electromagnetic simulations (EM), which are performed with Keysight ADS Momentum simulator [25]. Depending on the size of the training set, these simulations could last one/two weeks. However, these simulations are only performed once for a given fabrication technology, therefore being useful for several years, as technology nodes do not become obsolete in months. Any modeling technique can later be used in order to build a new model using the same training set.

\section{3) Model construction:}

This concerns the core functions used to build a surrogate model. Literature reports approaches based on artificial neural networks, support vector machines, parametric macromodels, Gaussian-process models, etc. [26]. In this work, ordinary Kriging models are used. Different Matlab toolboxes like SUMO [27] or DACE [28] support this type of models, being DACE the one used here. All the experiments in this paper use a zero-order polynomial regression model and a Gaussian correlation model parameter $\theta \in[0.01,10]$.

4) Model validation:

Many different techniques may be used in order to validate the model and assess its accuracy e.g. cross-validation, bootstrapping and subsampling [29]. In this work, in order to validate the model, a set of points was generated independently of the training samples. These samples will be referred to as test samples and were also generated using LHS. 
Table 1.

Average error (in \%) of inductance and quality factor for 560 test inductors: single model for all $N$.

\begin{tabular}{cc|cc|cc}
\hline \hline \multicolumn{2}{c|}{$20 \mathrm{MHz}$} & \multicolumn{2}{|c|}{$1 \mathrm{GHz}$} & \multicolumn{2}{c}{$2.5 \mathrm{GHz}$} \\
$\Delta \mathbf{L}_{(\%)}$ & $\Delta \mathbf{Q}_{(\%)}$ & $\Delta \mathbf{L}_{(\%)}$ & $\Delta \mathbf{Q}_{(\%)}$ & $\Delta \mathbf{L}_{(\%)}$ & $\Delta \mathbf{Q}_{(\%)}$ \\
\hline 0.04 & 0.27 & 0.52 & 0.79 & 17.75 & 3.21 \\
\hline \hline
\end{tabular}

\subsection{Proposed modeling strategy}

As shown in Section 2, inductance and quality factor are functions of the frequency. There have been attempts to build frequency-dependent models [30],[31] but they suffer from exponential complexity growth with the number of parameters and training samples. Problem-specific knowledge can be exploited to circumvent this problem. By looking at the typical $L / Q$ performances shown in Fig, 2 and the design goals introduced in Section 2, the optimization problem can be intelligently formulated to only require $L / Q$ modeling at a reduced number of frequency points, as will be detailed in this Section.

The initial strategy to build the model was to create a surrogate model valid in the complete design space using the entire training set. The main objective was to develop two different models (one for predicting inductance and another for quality factor) that were valid for inductors with any given number of turns $N$, inner diameter $D_{\text {in }}$ and turn width $w$. The technology selected was a $0.35 \mu \mathrm{m}$ CMOS technology, for which the process information required for EM simulation was available, and the variables were allowed to vary in the following ranges: $N \in[1,8], D_{i n} \in[10,300] \mu \mathrm{m}, w \in[5,25] \mu \mathrm{m}$, under the constraint that $D_{\text {out }}<400 \mu \mathrm{m}$. The spacing between turns $s$ was kept fixed at the minimum value $(s=2.5 \mu \mathrm{m})$ as no performance improvement is obtained for larger values. A parameterized cell developed by the authors, one instance of which is shown in Fig. 1, was used to generate the layout of the training and validation samples, which were then EM simulated. By using this strategy with 800 training inductors, the mean relative error for a test set with 560 inductor samples and three different frequencies is unacceptably large at higher frequencies, as it can be seen in Table 1.

By understanding that the number of inductor turns can only take some discrete values, e.g., in the implementation reported in this paper, it can only take integer values due to the parameterized cells used for EM simulation, it becomes clear that by creating several surrogate models, one for each number of turns (e.g. one model for inductors with two turns, another for inductors with three turns, etc.) the model accuracy can be increased. The generation of separate surrogate models for each number of turns instead of considering the number of turns as an input parameter of the surrogate model brings several benefits. Not only is the accuracy significantly enhanced but the computational cost is significantly decreased as the computational complexity of the training process grows exponentially with the number of samples. The number of models to create is manageable as the number of turns is typically between 1 and 8 .

This strategy increases the overall accuracy and efficiency of the model, as shown for the average relative errors of 560 test inductors in Table 2. However, some test inductors still present large $L$ and $Q$ errors, specifically at high frequencies for inductors with many turns. This can be explained by the fact that some inductors from the training set have their SRF below or around the frequency of operation. Gaussian process surrogate models assume continuity: if an input variable changes by a small amount, the output varies smoothly. However, this is not the case for the inductance if the training inductors have their $S R F$ close to the frequency of operation (see Fig. 2). Therefore, the use of these inductors in the model construction dramatically decreases the accuracy of the model for test inductors with $S R F$ above the frequency of operation. Potentially useful inductors have their $S R F$ sufficiently above the frequency of operation. The accuracy estimation of inductance and quality factor of these useful inductors is dramatically increased if only inductors with $S R F$ sufficiently above the operating frequency are used for model training. However, this option is only feasible if we can detect which inductors have their $S R F$ sufficiently above the frequency of operation and are, hence, useful.

Table 2.

Inductance and quality factor average error for 560 (70 for each number of turns) test inductors (in \%): one model for each $N$.

\begin{tabular}{c|cc|cc|cc}
\hline \hline \multirow{2}{*}{$\boldsymbol{N}$} & \multicolumn{2}{|c|}{$20 \mathrm{MHz}$} & \multicolumn{2}{c|}{$1 \mathrm{GHz}$} & \multicolumn{2}{c}{$2.5 \mathrm{GHz}$} \\
& $\Delta \mathbf{L}(\%)$ & $\Delta \mathbf{Q}_{(\%)}$ & $\Delta \mathbf{L}(\%)$ & $\Delta \mathbf{Q}_{(\%)}$ & $\Delta \mathbf{L}(\%)$ & $\Delta \mathbf{Q}_{(\%)}$ \\
\hline 1 & 0.09 & 0.42 & 0.16 & 0.66 & 0.15 & 0.81 \\
2 & 0.06 & 0.50 & 0.22 & 0.85 & 0.26 & 0.75 \\
3 & 0.03 & 0.40 & 0.14 & 0.73 & 0.19 & 0.51 \\
4 & 0.03 & 0.34 & 0.21 & 0.95 & 0.28 & 0.61 \\
5 & 0.02 & 0.21 & 0.18 & 0.83 & 0.55 & 0.69 \\
6 & 0.02 & 0.20 & 0.14 & 0.69 & 1.96 & 1.46 \\
7 & 0.01 & 0.23 & 0.10 & 0.43 & 10.73 & 1.47 \\
8 & 0.01 & 0.22 & 0.15 & 0.41 & 19.60 & 9.27 \\
\hline \hline
\end{tabular}


Therefore, in the proposed strategy, the construction of the model is based on a two-step method:

1) Generate surrogate models for the $S R F$ (for each number of turns) using all training inductors.

2) In order to generate highly accurate surrogate models for $L$ and $Q$, only those inductors from the training set whose $S R F$ is sufficiently above the operating frequency are used. For example, if the operating frequency is $2.5 \mathrm{GHz}$, only inductors with $S R F>3 \mathrm{GHz}$ are used to generate $L$ and $Q$ models. In Fig. 2, it is possible to observe that the selected inductor with eight turns is not useful at $2.5 \mathrm{GHz}$, since its $S R F<3 \mathrm{GHz}$.

Consequently, with this methodology, whenever a test inductor is going to be evaluated, its $S R F$ value is predicted first. If the predicted $S R F$ is below $3 \mathrm{GHz}$ the inductor is discarded since it is not useful for the selected operating frequency. Otherwise, its inductance and quality factor are calculated using the $L / Q$ models.

The algorithm for model training is, therefore, as follows:

\section{TRAINING PHASE}

\section{Step 1: Sampling:}

Generate set ITS of $B$ inductor training samples for each number of turns using LHS. Generate set $I V S$ of $B^{\prime}$ validation samples for each number of turns using LHS.

Step 2: Simulation:

Perform EM simulation of the $\left(B+B^{\prime}\right)$ samples

\section{Step 3: SRF modeling:}

For each number of turns $i=1, \cdots, N_{\max }$, generate model of self-resonance frequency using set ITS

Step 4: Inductor selection:

Extract from ITS the set of training inductors $I T S^{*}$ with $S R F>f_{o}+\Delta f$ where $f_{o}$ is the frequency of operation and $\Delta f$ is a safety margin.

\section{Step 5: L/Q modeling:}

For each number of turns $i=1, \cdots, N_{\max }$, generate $L / Q$ model at frequencies of interest using set $I T S^{*}$.

\section{Step 6: Validation:}

Use set IVS to validate the accuracy of the generated models.

Model training for a given technology and frequency of operation is performed only once. This model can be used for performance evaluation as many times as needed according to the following algorithm:

\section{EVALUATION PHASE}

For a set IES of $C$ inductors to evaluate $i=1, \cdots, C$ do:

\section{Step 1: $S R F$ evaluation}

Predict the self-resonance frequency of $i$-th inductor using the $S R F$ model.

\section{Step 2:}

If the predicted self-resonance frequency $S R F_{\text {pre }}>f_{o}+\Delta f$ go to Step 3. Otherwise, discard $i$-th inductor as a non-valid inductor for the desired frequency of operation and go to Step 1.

\section{Step 3: L/Q evaluation}

Predict $L$ and $Q$ of $i$-th inductor at frequencies of interest using the $L / Q$ model.

In order to evaluate the validity of the new proposed strategy, the 560 test inductors were evaluated for three different frequencies. The model errors for $L$ and $Q$ are shown in Table 3 and for the $S R F$ in Table 4. It can be concluded that by following

\begin{tabular}{|c|c|c|c|c|c|c|}
\hline \multirow[b]{2}{*}{$N$} & \multicolumn{2}{|c|}{$20 \mathrm{MHz}$} & \multicolumn{2}{|c|}{$1 \mathrm{GHz}$} & \multicolumn{2}{|c|}{$2.5 \mathrm{GHz}$} \\
\hline & $\Delta \mathbf{L}_{(\%)}$ & $\Delta \mathbf{Q}_{(\%)}$ & $\Delta \mathrm{L}_{(\%)}$ & $\Delta \mathbf{Q}_{(\%)}$ & $\Delta \mathbf{L}_{(\%)}$ & $\Delta \mathbf{Q}_{(\%)}$ \\
\hline 1 & 0.09 & 0.42 & 0.16 & 0.66 & 0.15 & 0.81 \\
\hline 2 & 0.06 & 0.50 & 0.22 & 0.85 & 0.26 & 0.75 \\
\hline 3 & 0.03 & 0.40 & 0.14 & 0.73 & 0.19 & 0.51 \\
\hline 4 & 0.03 & 0.34 & 0.21 & 0.95 & 0.28 & 0.61 \\
\hline 5 & 0.02 & 0.21 & 0.19 & 0.85 & 0.33 & 0.67 \\
\hline 6 & 0.02 & 0.24 & 0.16 & 0.79 & 0.35 & 0.49 \\
\hline 7 & 0.01 & 0.55 & 0.14 & 0.53 & 0.55 & 0.61 \\
\hline 8 & 0.02 & 0.33 & 0.06 & 0.33 & 0.34 & 0.88 \\
\hline
\end{tabular}


Table 4.

$S R F$ average error for 560 test inductors (in \%).

\begin{tabular}{llllllllll}
\hline \hline & $\boldsymbol{N}=\mathbf{1}$ & $\boldsymbol{N}=\mathbf{2}$ & $\boldsymbol{N}=\mathbf{3}$ & $\boldsymbol{N}=\mathbf{4}$ & $\boldsymbol{N}=\mathbf{5}$ & $\boldsymbol{N}=\mathbf{6}$ & $\boldsymbol{N}=\mathbf{7}$ & $\boldsymbol{N}=\mathbf{8}$ \\
\hline $\boldsymbol{S R F}$ & 1.28 & 1.61 & 0.69 & 0.58 & 0.35 & 0.34 & 0.38 & 0.34 \\
\hline \hline
\end{tabular}

this modeling strategy, the average error for inductance and quality factor is always below $1 \%$ for $L$ and $Q$ (at $2.5 \mathrm{GHz}$ ), which is the most accurate model for integrated inductors to the best of our knowledge.

For a better statistical assessment of the inductance and quality factor errors provided by the three strategies for surrogate modelling, box-and-whisker plots for each number of turns are shown in Fig. 4. To reduce the number of plots, these representations are limited to the inductance and quality factor at $2.5 \mathrm{GHz}$, the operating frequency, at which a high accuracy is expected to be more difficult to achieve. The column "All N" in each plot corresponds to a single model for all inductors with any number of turns (although the resulting error has been classified according to the number of turns to ease comparison with the other models), the column "NF" corresponds to a different model for each number of turns and finally, column "F" corresponds to the two-step surrogate modelling strategy in which inductors with $S R F$ lower than a specified limit are filtered

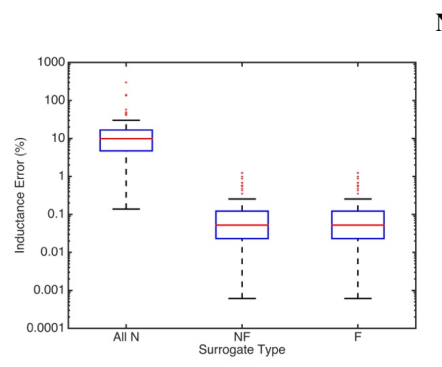

$\mathbf{N}=\mathbf{1}$
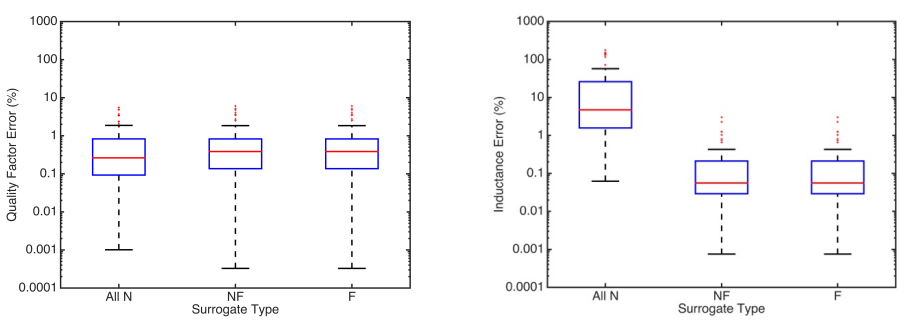

$\mathbf{N}=\mathbf{2}$

$\mathbf{N}=\mathbf{3}$
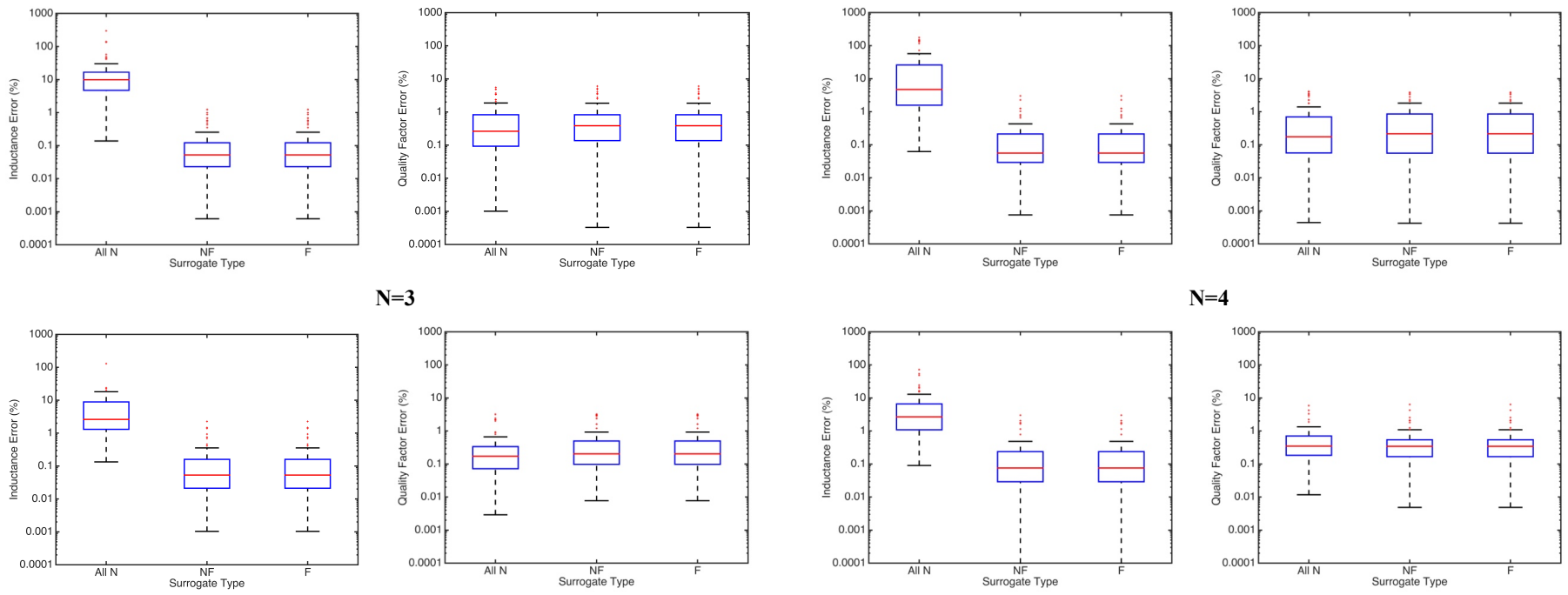

$\mathbf{N}=\mathbf{4}$

$\mathbf{N}=\mathbf{5}$
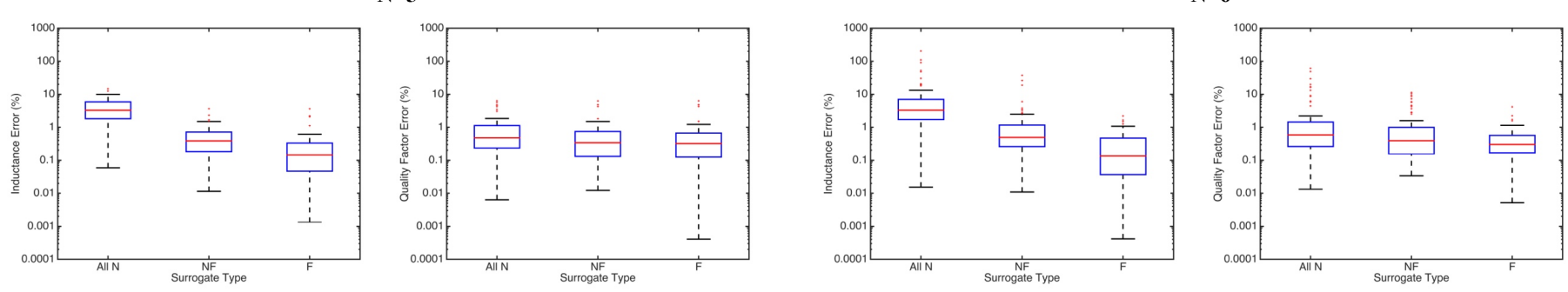

$\mathbf{N}=7$
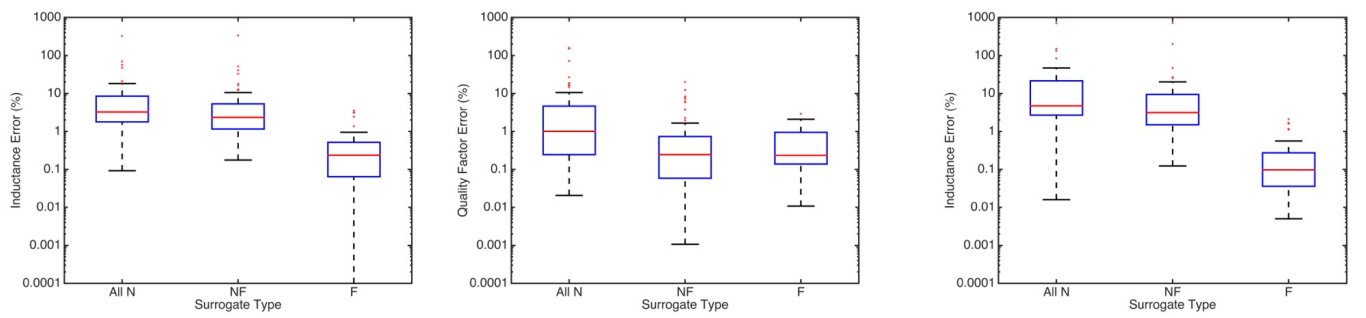

$\mathrm{N}=\mathbf{8}$

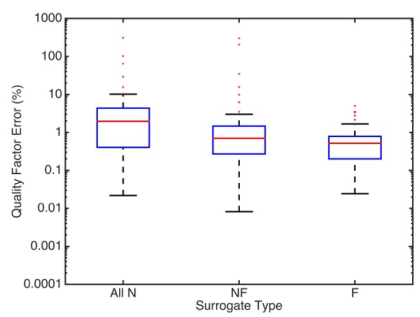

Fig. 4. Box-and-whisker plots of the relative error of inductance and quality factor for the test inductors with different number of turns. Three methods are compared: single model for all inductors, one model for each number of turns and the new two-step surrogate modeling strategy for each number of turns. 
out. Errors have been plotted using the same logarithmic scale for every plot for better comparison. As expected, a separate model for each number of turns yield a significant reduction of modelling errors, especially in inductance. This is important because, as the practical design experiments in Section 5 show, inductance accuracy is especially relevant. It can be noticed that the errors of the second and third model are the same for lower values of the number of turns $(N \leq 4)$. This is logical in this experiment because the $S R F$ usually decreases with the number of turns. For $N \leq 4$ the $S R F$ of every training and test inductor is sufficiently above $2.5 \mathrm{GHz}$ (the operating frequency) and, therefore, no inductor is being filtered out. However, for $N>4$, the errors with the two-step model (column "F") are considerably reduced, especially in inductance. It is not strange to find test inductors in conventional modeling strategies (columns "all N" and "NF") with errors well in excess of 10\% and the median of the inductance error lies between $1 \%$ and $10 \%$ for higher number of turns.

\section{Optimization Results and Comparisons}

\subsection{Single-Objective Optimization using PSO}

In this section, four different methodologies: EMO, OFFSO, ONSO and ONSO using expected improvement (for the sake of simplicity, further references to this method are denoted as ONSOEI) are applied to the synthesis of integrated inductors. The ECO methodology is not implemented since analytical models are not accurate at all, and therefore the optimization process yields suboptimal, and even invalid, inductors. The EMO method uses ADS Momentum as a performance evaluator and the OFFSO methodology uses a surrogate model, which was previously created using 800 training inductors $(B=100)$. The ONSO and ONSOEI methodologies have some differences when compared to the previous methods. The flow diagram of ONSO/ONSOEI is presented in Fig. 5. An initial surrogate coarse model is created using 40 (EM-simulated) training inductors. In this implementation of ONSO, the model is updated at each generation with the EM simulation results of the best individual of the current population. In ONSOEI, the model is updated by simulating the inductor that presents the highest expected improvement from the current population. However, if any of these individuals (either the best one from the current population or that with the highest expected improvement) had already been simulated and used for the model construction in previous iterations, this individual is not EM-simulated and the model does not have to be updated in that iteration.

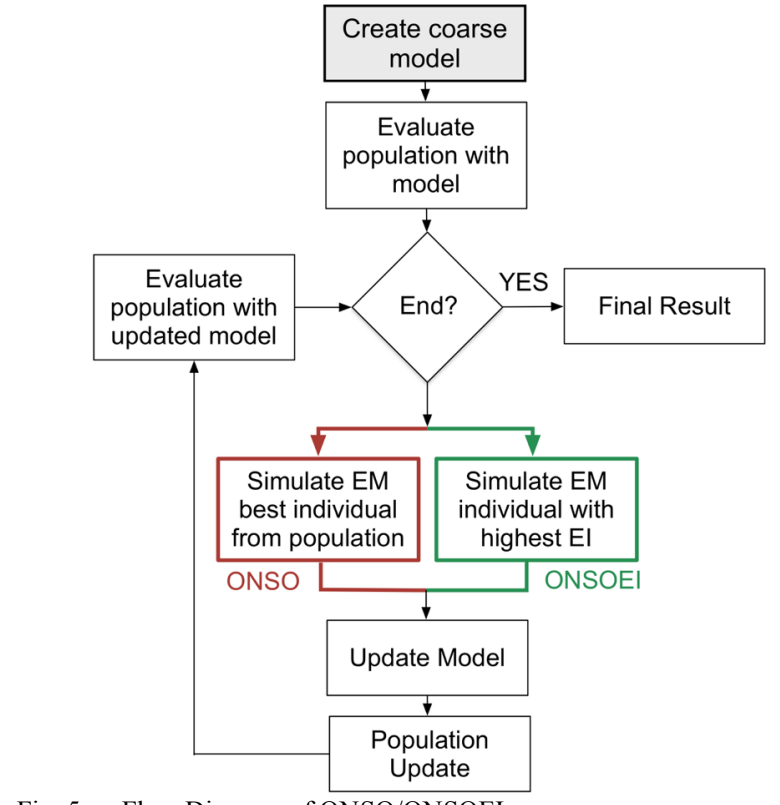

Fig. 5. Flow Diagram of ONSO/ONSOEI. 
Table 5 .

Inductor design variables

\begin{tabular}{cccc}
\hline \hline Parameter & Minimum & Step & Maximum \\
\hline$N$ & 1 & 1 & 8 \\
$w$ & $5 \mu \mathrm{m}$ & $0.05 \mu \mathrm{m}$ & $25 \mu \mathrm{m}$ \\
$D_{i n}$ & $10 \mu \mathrm{m}$ & $0.05 \mu \mathrm{m}$ & $300 \mu \mathrm{m}$ \\
$s$ & $2.5 \mu \mathrm{m}$ & $0.05 \mu \mathrm{m}$ & $2.5 \mu \mathrm{m}$ \\
\hline \hline
\end{tabular}

The technology used for inductor synthesis was the $0.35 \mu \mathrm{m}$ CMOS technology used in Section 4 . The bounds of both the optimization search space and the samples used to create the surrogate models are set by the design rules of the technology at the lower end, and by reasonable values at the upper end, and are shown in Table 5. The spacing is fixed to $2.5 \mu \mathrm{m}$. Inner diameter and turn width are forced to fit in a grid of $0.05 \mu \mathrm{m}$ due to fabrication technology constraints. All optimizations are performed with 40 particles and 200 generations.

Optimization constraints were defined to guarantee the good behavior of the inductor at the frequencies of interest. As stated in Section 2, these constraints are: inductance value is sufficiently flat from DC to slightly above the operating frequency and the self-resonance frequency is sufficiently above this frequency. The inductor must also fit within a $400 \mu \mathrm{mx} 400 \mu \mathrm{m}$ square. Since in the EMO methodology it is extremely expensive to accurately calculate the $S R F$ of the inductors, the latter constraint is approximated by imposing that the quality factor is near its maximum at the operating frequency and always with a positive slope around it [9]. Therefore, the optimization constraints are formulated as:

$$
\left\{\begin{array}{l}
\text { area }<400 \mu m \times 400 \mu m \\
\left|\frac{L_{\text {at } 2.5 \mathrm{GHz}}-L_{\text {at } 2.55 \mathrm{GHz}}}{L_{\text {at } 2.5 \mathrm{GHz}}}\right|<0.01 \\
\left|\frac{L_{\text {at } 2.5 \mathrm{GHz}}-L_{\text {at } 2.45 \mathrm{GHz}}}{L_{\text {at } 2.5 \mathrm{GHz}}}\right|<0.01 \\
\left|\frac{L_{\text {at } 2.5 \mathrm{GHz}}-L_{\text {at } 0.1 \mathrm{GHz}}}{L_{\text {at } 2.5 \mathrm{GHz}}}\right|<0.05 \\
Q_{\text {at } 2.55 \mathrm{GHz}}-Q_{\text {at } 2.5 \mathrm{GHz}}>0
\end{array}\right.
$$

To have a fair comparison with the other methods, and although it was not strictly necessary, the constraint related to the $S R F$ location was imposed identically in all methods.

The objective of the first test is to find an octagonal inductor with $L_{\text {spec }}=2 \mathrm{nH}$ at $2.5 \mathrm{GHz}$ while maximizing the quality factor. Since PSO is a single-objective algorithm, a weighted objective function was built so that the quality factor was maximized and the difference between the desired inductance and the one obtained by the algorithm was minimized:

$$
f(x)=-Q(x)+\lambda\left|L(x)-L_{\text {spec }}\right|
$$

where $\lambda$ was set equal to 5 .

Results for the best inductor obtained by a single execution of each methodology are shown in Table 6 . The performances of such optimal inductors have been electromagnetically simulated a posteriori, so that the $L$ and $Q$ values shown at the table can be fairly compared.

It must also be considered that the time data in Table 6 correspond to CPU time. The computer used for all simulations had two 6-core Intel ${ }^{\circledR}$ Xeon ${ }^{\circledR}$ E5-2630 v2 processors at $2.60 \mathrm{GHz}$, enabling parallelization of the evaluation of different solutions. Such parallelization is very effective for the EMO approach, achieving a reduction of elapsed time by about a $10 \mathrm{x}$ factor with respect to the CPU time. A priori, parallelization of the OFFSO approach is not worth given the total CPU times involved.

Table 6.

Results of one execution with all methods targeted at $L=2 \mathrm{nH}$ and maximizing $Q$.

Area $<400 \mu \mathrm{m} \times 400 \mu \mathrm{m}$

\begin{tabular}{cccccccc}
\hline \hline Method & $\boldsymbol{N}$ & $\begin{array}{c}\boldsymbol{D}_{\text {in }} \\
(\boldsymbol{\mu m})\end{array}$ & $\begin{array}{c}\boldsymbol{w} \\
(\boldsymbol{\mu m})\end{array}$ & \multicolumn{2}{c}{$\begin{array}{c}\text { Performances } \\
\text { @ 2.5GHz }\end{array}$} & $\begin{array}{c}\text { EM } \\
\text { sims. }\end{array}$ & \multirow{2}{*}{$\begin{array}{c}\text { CPU } \\
\text { time }\end{array}$} \\
\cline { 5 - 6 } & & & & $\boldsymbol{L}(\mathbf{n H})$ & $\boldsymbol{Q}$ & & \\
\hline EMO & 2 & 225 & 14.15 & 1.999 & 11.086 & 8000 & $106.5 \mathrm{~h}$. \\
ONSO & 2 & 225 & 14.10 & 1.999 & 11.073 & 45 & $32 \mathrm{~min}$. \\
ONSOEI & 2 & 225 & 14.10 & 1.999 & 11.073 & 55 & $56 \mathrm{~min}$. \\
OFFSO & 2 & 225 & 14.10 & 1.999 & 11.073 & 0 & $2 \mathrm{~min}$. \\
\hline \hline
\end{tabular}


Model updating in ONSO and ONSOEI is performed with at most one inductor at each iteration, and, therefore such parallelization is not directly possible.

Notice that the number of EM simulations, and, therefore, the computation times for the OFFSO, ONSO and ONSOEI methods only include the simulations performed during the execution of the PSO algorithm. Construction of the initial coarse model in ONSO and ONSOEI requires another 40 simulations ( 5 for each number of turns). This takes another 23.11 hours of CPU time. The CPU time required to simulate the 800 training samples in OFFSO amounts to 462.30 hours (around 19 days). Indeed, as stated above, parallelization of these evaluations in the machine with two 6-core processors reduces the elapsed time by approximately a factor $10 \mathrm{x}$ with respect to the CPU time.

Since the initial 40 EM simulations for the coarse model in ONSO and ONSOEI and the 800 samples for the OFFSO method can be run a priori and it is a one-time investment (they are independent of the required optimization objectives), they have not been included in the CPU time calculation of the optimization process shown in the table. The table only reflects the CPU time required to get the optimization results once the optimization goals are known. This means that the initial accurate simulations should not be accounted as time to build a given model as they are only performed once and can be used to build several different models using different techniques.

To understand the lack of proportionality between the CPU times above and those in the table, two considerations must be made:

1) Some simulations for the initial sampling take longer, since simulations of inductors with 8 turns require much more time than e.g., 3 turns, whereas convergence of the different methods in this example implies that most additional EM simulations correspond to inductors with less than 4 turns.

2) The EM simulations of the 40 samples of the coarse model in ONSO and ONSOEI and the 800 samples in OFFSO were performed for several hundreds of frequency points so that the results can be used for any synthesis problem independently of the frequencies of interest. If only the few frequency points necessary for this experiment were used, the CPU time is reduced from 23.11 hours to 1.22 hours, and from 462.30 hours to 24.36 hours respectively. However, in that case, new samples and their corresponding EM simulation are needed whenever a new frequency of operation is desired.

As an illustration example, the frequency behavior of the inductance and quality factor of the inductor obtained with the OFFSO methodology in the first test example (Table 6) are presented in Fig. 6 and they are compared against the EM simulation of the same inductor. The differences in inductance and quality factor of the OFFSO result and the EM simulation are also shown in the same figure. It is possible to observe that the accuracy of the model is remarkably good along all the frequency range. After the optimization process (which takes 2 minutes) the inductor synthesis is complete and the layout of this inductor is presented in Fig. 7.

The results for another experiment, this time addressing an inductor with $2.5 \mathrm{nH}$ and maximum quality factor at $2.5 \mathrm{GHz}$ are shown in Table 7.
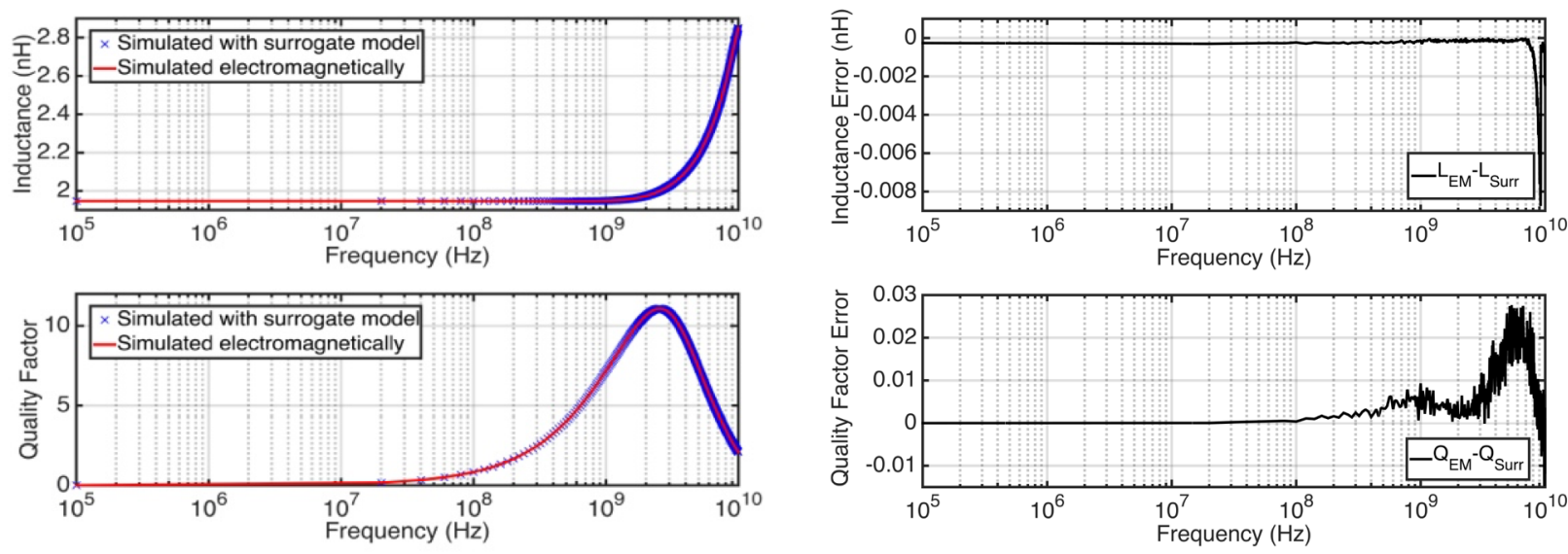

(a)

(b)

Fig. 6. Performance parameters of the $2 \mathrm{nH}$ inductor obtained in the first test example: (a) comparison of inductance and quality factor vs. frequency curves predicted by the surrogate model and those obtained with electromagnetic simulation and (b) absolute errors in inductance and quality factor. 
Table 7.

Results of one execution with all methods targeted at $L=2.5 \mathrm{nH}$ and maximizing $Q$.

Area $<400 \mu \mathrm{m} \times 400 \mu \mathrm{m}$.

\begin{tabular}{|c|c|c|c|c|c|c|c|}
\hline \multirow[t]{2}{*}{ "Method } & \multirow[t]{2}{*}{$\bar{N}$} & \multirow[t]{2}{*}{$\begin{array}{c}D_{\text {in }} \\
(\mu \mathrm{m})\end{array}$} & \multirow[t]{2}{*}{$\begin{array}{c}=w \\
(\mu \mathrm{m})\end{array}$} & \multicolumn{2}{|c|}{$\begin{array}{c}\text { Performances } \\
@ 2.5 \mathrm{GHz}\end{array}$} & \multirow[t]{2}{*}{$\begin{array}{c}\text { EM } \\
\text { sims. }\end{array}$} & \multirow[t]{2}{*}{$\begin{array}{l}\text { CPU } \\
\text { time }\end{array}$} \\
\hline & & & & $L(\mathrm{nH})$ & $Q$ & & \\
\hline EMO & 2 & 260 & 10.60 & 2.504 & 10.704 & 8000 & $111.2 \mathrm{~h}$. \\
\hline ONSO & 3 & 136 & 9.50 & 2.500 & 10.39 & 36 & $34.3 \mathrm{~min}$ \\
\hline ONSOEI & 2 & 259 & 10.35 & 2.499 & 10.713. & 59 & $67.6 \mathrm{~min}$ \\
\hline OFFSO & 2 & 260 & 10.80 & 2.498 & 10.742 & 0 & $2 \mathrm{~min}$ \\
\hline
\end{tabular}

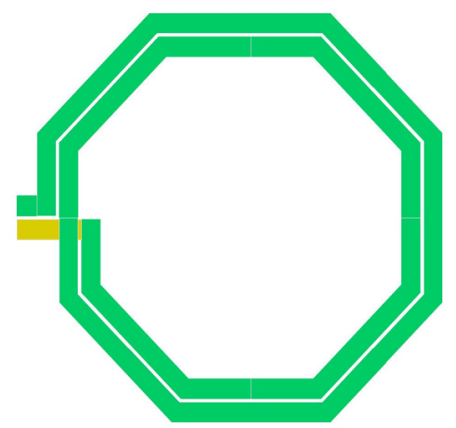

Fig. 7. Layout of the $2 \mathrm{nH}$ inductor obtained in the first test example.

As in any other computational intelligence algorithm, PSO also implies the introduction of randomness, and, hence, different runs may provide different results. Therefore, 20 independent runs were performed for each experiment. The mean values and standard deviations obtained with the different techniques are shown in Table 8 and Table 9.

From the comparison of these results of the different techniques for single-objective optimization, several conclusions can be drawn:

1) OFFSO converges to approximately the same solutions than the reference method EMO in all cases. However, the efficiency is drastically increased, since the CPU time decreases from more than 100 hours to roughly 2 minutes. These test examples demonstrate that the accuracy of the surrogate model used in the OFFSO methodology leads to the same design space areas with much higher efficiency.

2) All methods practically converge to the same solutions in the $2 \mathrm{nH}$ example but a larger variability between different executions appears for the $2.5 \mathrm{nH}$ example in ONSOEI and especially ONSO, yielding a slightly lower quality factor. This seems to indicate that despite the EM simulations used in order to iteratively increase the accuracy of ONSO, the accuracy of the initial coarse model is crucial for an optimal inductor synthesis, i.e. if during the first iterations, where the model is still quite inaccurate, the optimization algorithm leads to design space areas away from the optimal point, it is less likely that the optimization algorithm will converge to the global optimum. Moreover, the computation time rises to tens of minutes due to

Table 8.

Mean vaues and standard deviations (between brackets) with all methods targeted at $L=2 \mathrm{nH}$ and maximizing $Q$. Area $<400 \mu \mathrm{m} \times 400 \mu \mathrm{m}$

\begin{tabular}{ccccc}
\hline \hline \multirow{2}{*}{ Method } & \multicolumn{2}{c}{ Performances @2.5GHz } & EM sims. & $\begin{array}{c}\text { CPU } \\
\text { time }\end{array}$ \\
\cline { 2 - 3 } & $\boldsymbol{L}(\mathbf{n H})$ & $\boldsymbol{Q}$ & & $106.5(0.0) \mathrm{h}$. \\
EMO & $1.9992(0.0000)$ & $11.0861(0.0000)$ & $8000(0.0)$ & $32(0.0) \mathrm{min}$. \\
ONSO & $1.9990(0.0000)$ & $11.0731(0.0000)$ & $45(0.0)$ & $56(0.0) \mathrm{min}$. \\
ONSOEI & $1.9989(0.0004)$ & $11.0731(0.0005)$ & $55(0.0)$ & $2(0.0) \mathrm{min}$. \\
OFFSO & $1.9999(0.0006)$ & $11.0730(0.0004)$ & 0 & 0 \\
\hline \hline
\end{tabular}

Table 9.

Mean vaues and standard deviations (between brackets) with all methods targeted at $L=2.5 \mathrm{nH}$ and maximizing $Q$. Area $<400 \mu \mathrm{m} \times 400 \mu \mathrm{m}$

\begin{tabular}{|c|c|c|c|c|}
\hline \multirow[t]{2}{*}{ Method } & \multicolumn{2}{|c|}{ "Performances @2.5GHz } & \multirow[t]{2}{*}{ EM sims. } & \multirow{2}{*}{$\begin{array}{l}\mathrm{CPU} \\
\text { time }\end{array}$} \\
\hline & $L(\mathrm{nH})$ & $Q$ & & \\
\hline EMO & $2.502(0.001)$ & $10.650(0.102)$ & $8000(0.0)$ & $111.2(0.0) \mathrm{h}$. \\
\hline ONSO & $2.500(0.001)$ & $10.527(0.335)$ & $35.9(8.0)$ & 34.3 (8.3) $\mathrm{min}$. \\
\hline ONSOEI & $2.501(0.008)$ & $10.557(0.265)$ & $62.2(33.1)$ & $67.5(36.0) \mathrm{min}$ \\
\hline OFFSO & $2.496(0.010)$ & $10.648(0.144)$ & 0 & $2(0.0) \mathrm{min}$. \\
\hline
\end{tabular}


the additional EM simulations. The use of the expected improvement in ONSOEI improves in most cases the ONSO ability to locate the optimal regions of the search space and, therefore, the accuracy of the method is quite good. However, the use of this prescreening method brings a penalty in the form of additional EM simulations that typically rises the optimization time to about one hour.

In the examples shown above, the OFFSO, ONSO and ONSOEI methodologies converge to the same solutions either if the training inductors with $S R F$ close or below the frequency of operation are filtered out according to the two-step modeling method described above or not. Inductors with a number of turns $N \leq 3$ are obtained. All training inductors with these numbers of turns have a $S R F$ sufficiently above $2.5 \mathrm{GHz}$. Therefore, it becomes natural that the effect of the $S R F$ filtering methodology proposed in Section 4 is negligible.

However, when the specs are such that they are met with inductors with a larger number of turns, they tend to have smaller $S R F$ and the effect of the proposed modeling approach becomes more noticeable. To show this, we will consider the comparison to methods similar to OFFSO, ONSO and ONSOEI, but in which all inductors are used in the training phase of the L/Q model, i.e, no inductor is filtered out if its $S R F$ is close or below $2.5 \mathrm{GHz}$. Correspondingly, we will denote these methods as OFFSO_NF, ONSO_NF and ONSOEI_NF.

In a first example, an inductor of $2.9 \mathrm{nH}$ with maximum quality factor that fits into a $125 \mu \mathrm{m} \times 125 \mu \mathrm{m}$ square is requested. Identical performance constraints to the previous examples are imposed. The results for one execution are shown in Table $10^{1}$. In this case, OFFSO and OFFSO_NF arrive at different results. Moreover, when the resulting inductors are EM-simulated, the performance constraints in OFFSO_NF are not met any more. ONSO, ONSOEI and ONSOEI_NF arrive at results similar to OFFSO, but ONSO_NF is unable to converge to a feasible solution (as indicated in the fifth column in Table 10). A second example is shown in Table $11^{2}$. In this case an inductance of $4.6 \mathrm{nH}$ with maximum quality factor within a $140 \mu \mathrm{mx} 140 \mu \mathrm{m}$ square is specified. In this case, all methods but EMO and OFFSO are unable to converge to a solution due to the larger errors of the surrogate models of inductance and quality factor.

As in the previous experiments, 20 executions of each algorithm were performed for both cases. The statistical analysis of the

Table 10.

Results of one execution with all methods targeted at $L=2.9 \mathrm{nH}$ and maximizing $Q$. Area $<125 \mu \mathrm{m} \times 125 \mu \mathrm{m}$.

\begin{tabular}{|c|c|c|c|c|c|c|c|c|c|}
\hline & \multirow[t]{2}{*}{$N$} & \multirow[t]{2}{*}{$\begin{array}{c}D_{i n} \\
(\mu \mathrm{m})\end{array}$} & \multirow[t]{2}{*}{$\begin{array}{c}w \\
(\mu \mathrm{m})\end{array}$} & \multirow{2}{*}{  } & \multicolumn{2}{|c|}{$\begin{array}{l}\text { Performances } \\
@ 2.5 \mathrm{GHz}\end{array}$} & \multirow{2}{*}{ 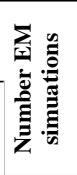 } & \multirow{2}{*}{ 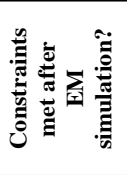 } & \multirow[t]{2}{*}{$\begin{array}{l}\text { CPU } \\
\text { time }\end{array}$} \\
\hline & & & & & $L(\mathrm{nH})$ & $Q$ & & & \\
\hline EMO & 6 & 36 & 5.15 & yes & 2.90 & 8.46 & 8000 & yes & $418.5 \mathrm{~h}$. \\
\hline ONSO & 6 & 35 & 5.45 & yes & 2.89 & 8.49 & 9 & yes & $32.2 \mathrm{~min}$. \\
\hline ONSO_NF & 4 & 70 & 5.00 & no & - & - & 2 & - & $4.2 \mathrm{~min}$. \\
\hline ONSOEI & 6 & 35 & 5.45 & yes & 2.89 & 8.49 & 45 & yes & $111.2 \mathrm{~min}$. \\
\hline ONSOEI_NF & 6 & 35 & 5.45 & yes & 2.89 & 8.49 & 45 & yes & $111.2 \mathrm{~min}$. \\
\hline OFFSO & 6 & 37 & 5.3 & yes & 2.98 & 8.55 & 0 & yes & $2 \mathrm{~min}$. \\
\hline OFFSO_NF & 6 & 40 & 5.00 & yes & 3.11 & 8.59 & 0 & no & $2 \mathrm{~min}$. \\
\hline
\end{tabular}

Table 11.

Results of one execution with all methods targeted at $L=4.6 \mathrm{nH}$ and maximizing $Q$. Area $<140 \mu \mathrm{m} \times 140 \mu \mathrm{m}$.

\begin{tabular}{|c|c|c|c|c|c|c|c|c|c|}
\hline & \multirow[t]{2}{*}{$\overline{N N}$} & \multirow[t]{2}{*}{$\begin{array}{c}D_{i n} \\
(\mu \mathrm{m})\end{array}$} & \multirow[t]{2}{*}{$\begin{array}{c}w \\
(\mu \mathrm{m})\end{array}$} & \multirow{2}{*}{ 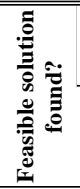 } & \multicolumn{2}{|c|}{$\begin{array}{l}\text { Performances } \\
\text { @ 2.5GHz }\end{array}$} & \multirow{2}{*}{ 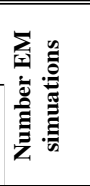 } & \multirow{2}{*}{  } & \multirow[t]{2}{*}{$\begin{array}{l}\text { CPU } \\
\text { time }\end{array}$} \\
\hline & & & & & $L(\mathrm{nH})$ & $Q$ & & & \\
\hline EMO & 7 & 40 & 5.05 & yes & 4.56 & 8.616 & 8000 & yes & $590.5 \mathrm{~h}$. \\
\hline ONSO & 5 & 70 & 5 & no & - & - & 3 & - & $6.8 \mathrm{~min}$. \\
\hline ONSO_NF & 5 & 70 & 5 & no & - & - & 3 & - & $7.5 \mathrm{~min}$. \\
\hline ONSOEI & 6 & 55 & 5 & no & - & - & 5 & - & $12.4 \mathrm{~min}$. \\
\hline ONSOEI_NF & 5 & 70 & 5 & no & - & - & 9 & - & $11.4 \mathrm{~min}$. \\
\hline OFFSO & 7 & 40 & 5 & yes & 4.54 & 8.520 & 0 & yes & $2 \mathrm{~min}$ \\
\hline OFFSO_NF & 6 & 55 & 5.05 & no & - & - & 0 & - & $1.5 \mathrm{~min}$. \\
\hline
\end{tabular}

\footnotetext{
${ }^{1}$ The elapsed time in EMO reduces by a factor 10 with respect to the CPU time when all cores of the twin 6-core processor are used.

${ }^{2}$ The elapsed time in EMO reduces by a factor 10 with respect to the CPU time when all cores of the twin 6-core processor are used.
} 
Table 12.

Mean vaues and standard deviations (between brackets) with all methods targeted at $L=2.9 \mathrm{nH}$ and maximizing $Q$. Area $<125 \mu \mathrm{m} \times 125 \mu \mathrm{m}$.

\begin{tabular}{|c|c|c|c|c|c|c|}
\hline & \multirow{2}{*}{ 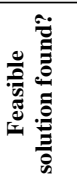 } & \multicolumn{2}{|c|}{$\begin{array}{l}\text { Performances } \\
@ 2.5 \mathrm{GHz}\end{array}$} & \multirow{2}{*}{ 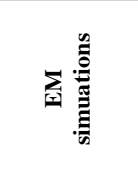 } & \multirow{2}{*}{ 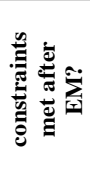 } & \multirow[t]{2}{*}{$\begin{array}{l}\text { CPU } \\
\text { time }\end{array}$} \\
\hline & & $L(\mathrm{nH})$ & $\bar{Q}$ & & & \\
\hline EMO & $20 / 20$ & $2.904(0.000)$ & $8.460(0.000)$ & $8000(0.0)$ & $20 / 20$ & $418.5(0.0) \mathrm{h}$. \\
\hline ONSO & $11 / 20$ & $2.891(0.000)$ & $8.488(0.000)$ & $9.33(6.02)$ & $11 / 20$ & $32.2(0.0) \mathrm{min}$. \\
\hline ONSO_NF & $0 / 20$ & - & - & $2(0.0)$ & $0 / 20$ & $4.2(0.0) \mathrm{min}$. \\
\hline ONSOEI & $20 / 20$ & $2.890(0.002)$ & $8.483(0.022)$ & $45(0.0)$ & $20 / 20$ & $111.2(0.0) \mathrm{min}$. \\
\hline ONSOEI_NF & $13 / 20$ & $2.889(0.004)$ & $8.422(0.182)$ & $37.1(15.1)$ & $13 / 20$ & 95.2 (37.5) $\mathrm{min}$. \\
\hline OFFSO & $20 / 20$ & $2.986(0.004)$ & $8.545(0.018)$ & 0 & $20 / 20$ & $2(0.0) \mathrm{min}$. \\
\hline OFFSO_NF & $20 / 20$ & $3.110(0.000)$ & $8.590(0.000)$ & 0 & $0 / 20$ & $1.5(0.0) \mathrm{min}$. \\
\hline
\end{tabular}

Table 13.

Mean vaues and standard deviations (between brackets) with all methods targeted at $L=4.6 \mathrm{nH}$ and maximizing $Q$. Area $<140 \mu \mathrm{m} \times 140 \mu \mathrm{m}$

\begin{tabular}{|c|c|c|c|c|c|c|}
\hline & \multirow{2}{*}{ 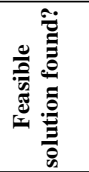 } & \multicolumn{2}{|c|}{$\begin{array}{c}\text { Performances } \\
@ 2.5 \mathrm{GHz}\end{array}$} & \multirow{2}{*}{ 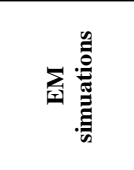 } & \multirow{2}{*}{ 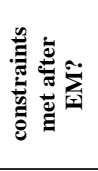 } & \multirow[t]{2}{*}{$\begin{array}{l}\text { CPU } \\
\text { time }\end{array}$} \\
\hline & & $L(\mathrm{nH})$ & $Q$ & & & \\
\hline EMO & $20 / 20$ & $4.562(0.017)$ & $8.564(0.131)$ & $8000(0.0)$ & $20 / 20$ & $590.5(0.0) \mathrm{h}$. \\
\hline ONSO & $0 / 20$ & - & - & $4.15(2.5)$ & $0 / 20$ & 9.4 (4.4) $\mathrm{min}$. \\
\hline ONSO_NF & $0 / 20$ & - & - & $3(0.0)$ & $0 / 20$ & $7.1(0.4) \mathrm{min}$. \\
\hline ONSOEI & $0 / 20$ & - & - & $5(0.0)$ & $0 / 20$ & $12.4(0.0) \mathrm{min}$. \\
\hline ONSOEI_NF & $0 / 20$ & - & - & $6.8(2.0)$ & $0 / 20$ & $12.0(0.5) \mathrm{min}$ \\
\hline OFFSO & $20 / 20$ & $4.540(0.007)$ & $8.530(0.029)$ & 0 & $20 / 20$ & $2(0.0) \mathrm{min}$. \\
\hline OFFSO_NF & $0 / 20$ & - & - & 0 & $0 / 20$ & $1.5(0.0) \mathrm{min}$. \\
\hline
\end{tabular}

results is shown in Table 12 and Table $13^{3}$. The second column in these tables shows how many of the 20 executions the optimization algorithm found a feasible solution. The statistical analysis of $L$ and $Q$ has been performed only for the feasible solutions found. It can be checked in Table 12 that ONSOEI arrives to similar solutions than OFFSO, although with a penalty in the computation time. ONSO_NF never arrives at a feasible solution, like in the single execution shown in Table 10. It is also found that ONSO and ONSOEI_NF only converge to a solution about half of the executions. Table 13 shows that only EMO and the proposed OFFSO approach always converge to a solution and they are quite similar, but with orders of magnitude less computational effort in the OFFSO case.

From the latter experiments, it can be concluded that in many cases the proposed two-step surrogate modeling strategy plays a key role in the proper convergence and accuracy of the surrogate-based optimization techniques for inductor synthesis.

\subsection{Multi-objective optimization using NSGA-II}

One of the advantages of having a fast and accurate surrogate model that needs no EM simulations during the optimization process is the ability of using this model within a multi-objective optimization algorithm.

Given the lower maturity of ONSO-like multi-objective optimization techniques and the fact that already for single-objective optimization of inductors, the ONSO and ONSOEI methodologies have not provided better solutions than the OFFSO approach and always with a higher computation time, this section will compare the OFFSO and EMO methodologies with multi-objective optimization algorithms. The effect of the two-step modeling approach will be also studied.

The search space is the same used in the previous optimizations and shown in Table 5. As a first test example, an optimization with two objectives was performed. The optimization problem was formulated as:

\footnotetext{
${ }^{3}$ The elapsed time in EMO reduces by a factor 10 with respect to the CPU time when all cores of the twin 6-core processor are used.
} 
maximize $L_{\text {at } 2.5 \mathrm{GHz}}$

maximize $Q_{\text {at } 2.5 \mathrm{GHz}}$

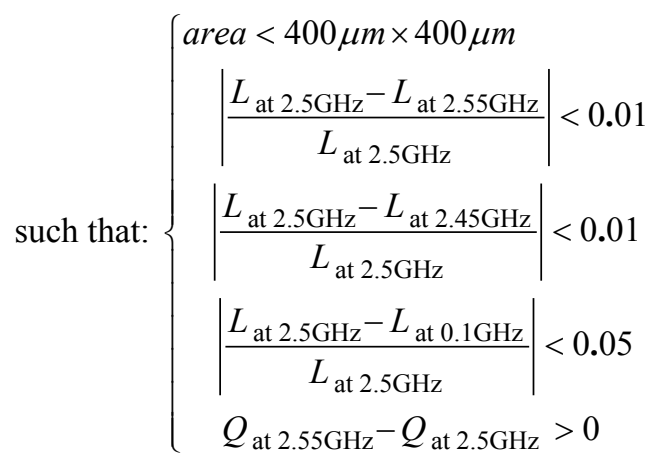

This optimization was performed with 300 individuals and 100 generations. The results are presented in Fig. 8, where the results of the optimization using the surrogate model have been electromagnetically simulated so that the accuracy can be fairly compared. It is possible to observe that the Pareto fronts obtained by both methods are very similar. The advantage of using OFFSO is the efficiency. While with EMO the optimization lasted 355.55 hours (around 15 days CPU time) ${ }^{4}$, the OFFSO method lasted 4 minutes, which is an increase in efficiency of about three orders of magnitude.

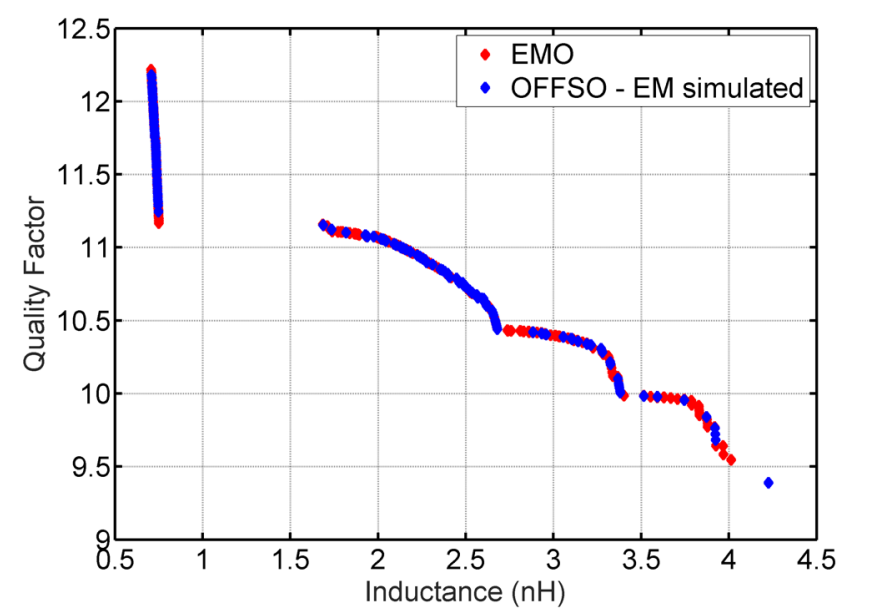

Fig. 8. Pareto-optimal front for a two objective optimization, maximizing quality factor and inductance.

A second test example was an optimization with 3 objectives, this time with 1000 individuals and 80 generations. Since area is of great importance especially in IC technologies, area minimization was added as a third objective in eq.(6). A major motivation for selecting a large population size is that the application of inductor fronts to RF circuit design benefit from denser Pareto fronts [19]. The results are shown in Fig. 9. The results of the surrogate model that are shown in Fig. 9 have been electromagnetically simulated a posteriori so that both fronts can be fairly compared.

Again, the Pareto fronts achieved by both methods are very similar. A more accurate comparison can be performed by using well-known performance metrics. From the bunch of performance metrics, we have selected two: hypervolume and set coverage [32].

Hypervolume is calculated as the union of the hypercubes determined by each solution in the objective space and a reference point. The hypervolume metric accounts for convergence and diversity of the Pareto front. A feature of the hypervolume metric is that it does not require any knowledge of the true Pareto front, especially convenient in our engineering problem in which the true Pareto front is not known. As our goal is to compare the Pareto fronts generated with two different techniques, and the hypervolume metric depends on the selected reference point, the same reference point is used in both cases.

Given two solution sets, $P_{1}$ and $P_{2}$, the set coverage is defined as:

${ }^{4}$ The elapsed time reduces to 29 hours with respect to the CPU time when all cores of the twin 6-core processor are used. 
$C\left(P_{1}, P_{2}\right)=\frac{\left|\left\{b \in P_{2} \mid \exists a \in P_{1}, a \quad b\right\}\right|}{\left|P_{2}\right|}$

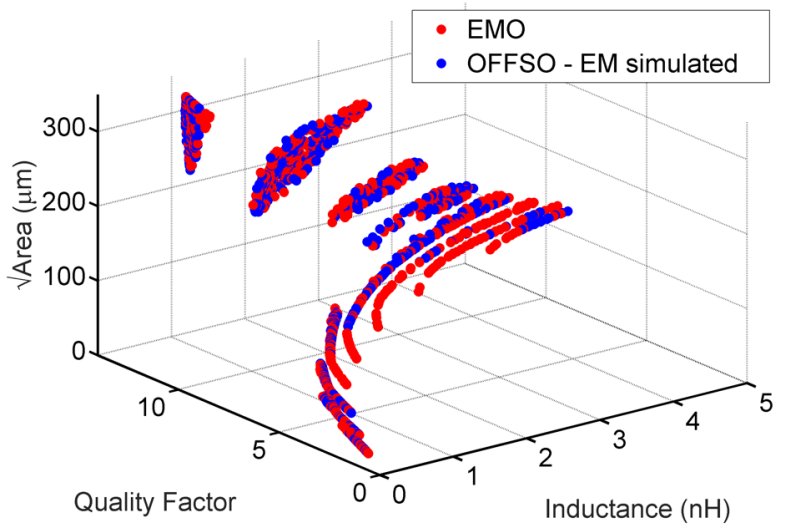

Fig. 9. EM simulation of Pareto-optimal fronts for a three objective optimization, maximization of quality factor and inductance and minimization of area using EMO and OFFSO methods.

where $a \quad b$ means that $a$ dominates $b$. The motivation to use set coverage is that allows performing binary comparisons between two fronts as the percentage of points of each front dominated by the other one are determined. A similar value of $C\left(P_{1}, P_{2}\right)$ and $C\left(P_{2}, P_{1}\right)$ implies that no front is better than the other.

The hypervolume of the Pareto front obtained with the EMO approach was $H V_{E M O}=10674$ and that with the OFFSO approach (after electromagnetically simulating the final results) was $H V_{\text {OFFSO }}=10604$, which is very similar. Regarding the set coverage, the calculated figures were:

$$
\begin{aligned}
& C\left(P F_{E M}, P F_{S U R R}\right)=0.16 \\
& C\left(P F_{\text {SURR }}, P F_{E M}\right)=0.14
\end{aligned}
$$

that indicates that practically the same percentage of points are dominated by the other front.

The EMO optimization took 1926.39 hours (roughly 80 days CPU time) $)^{5}$ while the OFFSO approach lasted 7 minutes, which is an incredible increase in efficiency while obtaining very similar Pareto fronts due to the accuracy of the surrogate model.

It is also interesting to compare the results with those of the optimization using the surrogate model without the first filtering stage, i.e., the OFFSO_NF methods. Fig. 10 compares the results of the EMO approach and the OFFSO_NF approach (after electromagnetically simulating the final results). There are areas of the Pareto front that are not found with the surrogate-based optimization (infeasible points are not plotted). These areas mostly correspond to inductors with larger number of turns, where

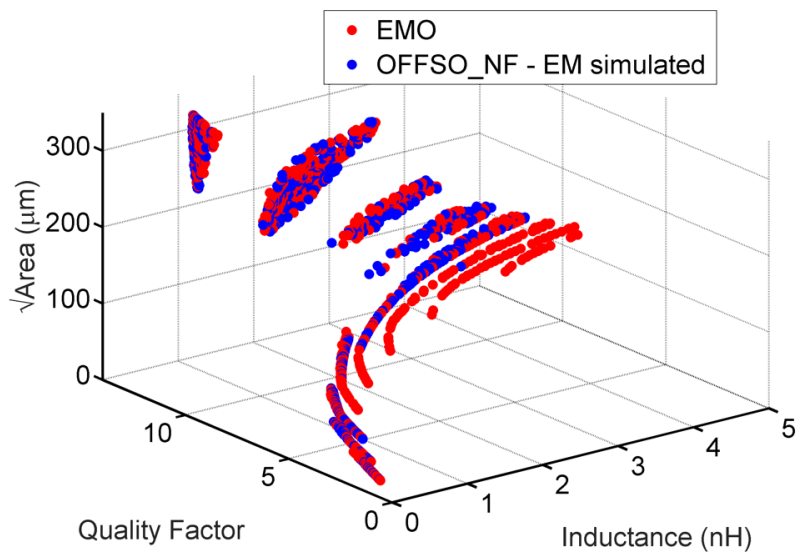

Fig. 10. EM simulation of Pareto-optimal front for a three objective optimization, maximization of quality factor and inductance and minimization of area using EMO and OFFSO_NF methods.

${ }^{5}$ The elapsed time reduces to 144 hours when all cores of the twin 6-core processor are used. 
the $S R F$ is considerably lower and the $S R F$ filtering approach becomes more noticeably for the $2.5 \mathrm{GHz}$ operating frequency.

The hypervolume of the Pareto front obtained now with the OFFSO_NF approach (after electromagnetically simulating the final results) was $H V_{O_{F F S O} N F}=9626$, which is clearly inferior to the EMO and OFFSO approaches. Regarding the set coverage, the calculated figures were:

$$
\begin{aligned}
& C\left(P F_{E M}, P F_{\text {SURR }}\right)=0.22 \\
& C\left(P F_{S U R R}, P F_{E M}\right)=0.13
\end{aligned}
$$

that indicates that the quality of the surrogate model has clearly decreased.

\section{Conclusions}

The application of Gaussian-process surrogate modeling strategies has been explored to enhance the efficiency-accuracy tradeoff of conventional analytical or EM-based inductor optimization techniques for RF integrated circuits. A new two-step surrogate modeling strategy has been proposed that dramatically improves the model accuracy. Implementation of the surrogate modeling methodology for another inductor topology or another fabrication technology just implies generating the corresponding parameterized cell and the electromagnetic simulation of a new set of training inductors. This task must be performed only once and implies just a few hours of user preparatory effort and a few weeks of CPU time (few days elapsed time in multi-core computers) for simulation.

It has been found that when prescreening techniques are used in surrogate-assisted optimization techniques, similar results to EM-based approaches have been obtained in most cases with a significantly lower computation time. However, it is also found that similar or better results are obtained if offline surrogate models with a sufficient number of inductor samples are created following the proposed modeling strategy. Much lower CPU times must be invested during the optimization process since expensive EM simulations are not performed during the optimization stage. The quality of the results of the proposed two-step surrogate modeling strategy is also competitive in multi-objective optimization of integrated inductors, whereas orders of magnitude computation time is saved.

\section{Acknowledgements}

Funding: This work was supported in part by the Spanish Ministry of Economy, Industry and Competitiveness and ERDF (grants TEC2013-45638-C3-3-R and TEC2016-75151-C3-3-R); and by Junta de Andalucía (grant P12-TIC-1481).

\section{References}

[1] R. A. Rutenbar, G. G. E. Gielen, and J. Roychowdhury, "Hierarchical Modeling, Optimization, and Synthesis for System-Level Analog and RF Designs," Proceedings of the IEEE, vol. 95, pp. 640-669, 2007.

[2] A. Niknejad, Electromagnetics for High-Speed Analog and Digital Communication Circuits: Cambridge University Press, 2007.

[3] https://www.cadence.com/content/cadence-www/global/en_US/home/tools/custom-ic-analog-rf-design/circuit-simulation/spectre-rf-option.html [Accessed March 1, 2017].

[4] https://www.synopsys.com/tools/Verification/AMSVerification/CircuitSimulation/HSPICE/Pages/default.aspx [Accessed July 31, 2016].

[5] F. Passos et al., "Lumped Element Model for Arbitrarily Shaped Integrated Inductors: A Statistical Analysis," in IEEE International Conference on Microwaves, Communications, Antennas and Electronic Systems, pp. 1-5, 2013.

[6] C. Wang, H. Liao, C. Li, R. Huang, W. Wong, X. Zhang, and Y. Wang, "A Wideband Predictive Double- $\pi$ Equivalent-Circuit Model for On-Chip Spiral Inductors," in IEEE Transactions on Electron Devices, vol. 56, no. 4, pp. 609-619, 2009.

[7] Y. Cao et al., "Frequency-Independent Equivalent Circuit Model for On-Chip Spiral Inductors," in IEEE Journal of Solid-State Circuits, vol. 38, no. 3, pp. 419-426, 2003.

[8] A. I. J. Forrester, A. Sobester, and A. J. Keane, Engineering Design via Surrogate Modelling - A Practical Guide. Wiley, 2008.

[9] R. González-Echevarría, et al, "Automated Generation of the Optimal Performance Trade-Offs of Integrated Inductors," in IEEE Transactions on Computer-Aided Design of Integrated Circuits and Systems, vol.33, no.8, pp. 1269-1273, 2014.

[10] F. Passos et al., "Physical vs. Surrogate Models of Passive RF devices," in Proceedings IEEE International Symposium on Circuits and Systems (ISCAS), pp.117-120, 2015.

[11] S.K. Mandal, S. Sural, A. Patra, "ANN- and PSO-Based Synthesis of On-Chip Spiral Inductors for RF ICs," in IEEE Transactions on Computer-Aided Design of Integrated Circuits and Systems, vol.27, no.1, pp.188-192, 2008.

[12] I. Couckuyt, F. Declercq, T. Dhaene, H. Rogier and L. Knockaert, "Surrogate-Based Infill Optimization Applied to Electromagnetic Problems", in International Journal of RF and Microwave Computer-Aided Engineering, vol. 20, no. 5, pp. 492-501, 2010.

[13] M. Ballicchia and S. Orcioni, "Design and Modeling of Optimum Quality Spiral Inductors with Regularization and Debye Approximation," IEEE Transactions on Computer-Aided Design of Integrated Circuits and Systems, vol.29, no.11, pp.1669-1681, Nov. 2010

[14] B. Liu, G. Gielen and F.V. Fernandez, Design Automation of Analog and High-Frequency Circuits: A Computational Intelligence Approach, Springer, 2013.

[15] C. T. Carrasco, C. J. Sieiro, J. M. Lopez-Villegas, N. Vidal, R. Gonzalez-Echevarría, and M. E. Roca, "Mixed-Mode Impedance and Reflection Coefficient of Two-Port Devices," Progress In Electromagnetics Research, Vol. 130, 411-428, 2012.

[16] J. Kennedy and R. Eberhart, "Particle swarm optimization," in Proceedings IEEE International Conference on Neural Networks, vol.4, pp. 1942-1948, 1995.

[17] K. Deb, A. Pratap, S. Agarwal, and T. Meyarivan, "A Fast and Elitist Multiobjective Genetic Algorithm: NSGA-II," IEEE Transactions on Evolutionary Computation, vol. 6, pp. 182-197, 2002. 
[18] K. Deb, "An Efficient Constraint Handling Method for Genetic Algorithms," Computer Methods in Applied Mechanics and Engineering, vol. 186, pp. 311$338,2000$.

[19] R. González-Echevarría et al., "An Automated Design Methodology of RF Circuits by Using Pareto-Optimal Fronts of EM-Simulated Inductors," in IEEE Transactions on Computer-Aided Design of Integrated Circuits and Systems, DOI: 10.1109/TCAD.2016.2564362.

[20] D.R. Jones, M. Schonlau and W.J. Welch, "Efficient Global Optimization of Expensive Black-Box Functions," in Journal of Global Optimization, vol. 13, pp. 455-492, 1998.

[21] I. Paenke, J. Branke and Y. Jin, "Efficient Search for Robust Solutions by Means of Evolutionary Algorithms and Fitness Approximation," in IEEE Transactions on Evolutionary Computation, vol. 10, no. 4, pp. 405-420, 2006.

[22] J. Knowles, "ParEGO: A Hybrid Algorithm With On-Line Landscape Approximation for Expensive Multi-objective Optimization Problems, “ in IEEE Transactions on Evolutionary Computation, vol. 10, no. 1, pp. 50-66, 2006.

[23] M.T.M. Emmerich, K.C. Giannakoglou and B. Naujoks, "Single- and Multi-objective Evolutionary Optimization Assisted by Gaussian Random Field Metamodels," in IEEE Transactions on Evolutionary Optimization, Vol. 10, No. 4, pp. 421-439, 2006.

[24] Q. Zhang, W. Liu, E. Tsang and B. Virginas, "Expensive Multi-objective Optimization by MOEA/D with Gaussian Process Model," in IEEE Transactions on Evolutionary Computation, vol. 14, no. 3, pp. 456-474, 2010.

[25] http://www.keysight.com/en/pc-1887116/momentum-3d-planar-em-simulator [Accessed July 31, 2016].

[26] M. B. Yelten, T. Zhu, S. Koziel, P. D. Franzon and M. B. Steer, "Demystifying Surrogate Modeling for Circuits and Systems," in IEEE Circuits and Systems Magazine, vol. 12, no. 1, pp. 45-63, 2012.

[27] D. Gorissen, I. Couckuyt, P. Demeester, T. Dhaene, and K. Crombecq. "A Surrogate Modeling and Adaptive Sampling Toolbox for Computer Based Design," The Journal of Machine Learning Research, vol. 11, pp. 2051-2055, 2010.

[28] http://www2.imm.dtu.dk/projects/dace/ [Accessed March 1, 2017].

[29] B. Bischl, O. Mersmann, H. Trautmann and C. Weihs, "Resampling Methods for Meta-Model Validation with Recommendations for Evolutionary Computation", Evolutionary Computation, vol. 20, No. 2, pp. 249-275, 2012.

[30] F. Ferranti, L. Knockaert, T. Dhaene and G. Antonini, "Parametric Macromodeling based on Amplitude and Frequency Scaled Systems with Guaranteed passivity" in International Journal of Numerical Modelling: Electronic Networks, Devices and Fields, vol. 25, no. 2, pp. 139-151, 2012.

[31] F. Passos, E. Roca, R. Castro-López, F.V. Fernández, Y. Ye, D. Spina and T. Dhaene, "Frequency-dependent parameterized macromodeling of integrated inductors," Proc. $13^{\text {th }}$ Int. Conference on Synthesis, Modeling, Analysis and Simulation Methods and Applications to Circuit Design (SMACD), June 2016.

[32] E. Zitzler and L. Thiele, "Multiobjective evolutionary algorithms: a comparative case study and the Strength Pareto Approach," IEEE Transactions on Evolutionary Computation, vol. 3, pp. 257-271, Nov. 1999.



F. Passos was born in Lisbon in 1989. He received the B.S. and M.S. degrees from the New University of Lisbon, in 2011 and 2013 respectively. Fábio is currently pursuing his $\mathrm{PhD}$ degree from University of Seville. He is conducting his work at the Institute of Microelectronics of Seville (IMSE-CNM-CSIC). He received an outstanding paper award in MIXDES 2013 and was the SMACD 2016 Electronic Design Automation (EDA) competition winner. His research interests include the modeling of passive RF devices, computer-aided design (CAD) and design methodologies for analog and RF circuits.

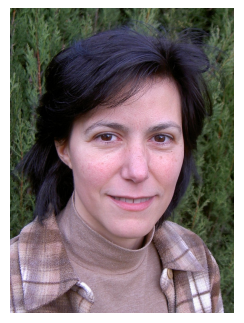

E. Roca received the physics and the Ph.D. degrees from the University of Barcelona, Spain, in 1990 and 1995, respectively. From November 1990 to April 1995, she worked at IMEC, Leuven, Belgium, in the field of infrared detection aiming to obtain large arrays of CMOS compatible silicide Schottky diodes. Since 1995, she has been with the Institute of Microelectronics of Seville, (IMSE-CNM-CSIC), Spain, where she holds the position of Tenured Scientist. Her research interests lie in the field of modeling and design methodologies for analog, mixed-signal and RF integrated circuits. She has been involved in several research projects with different institutions: Commission of the EU, ESA, ONR-NICOP, etc. She has also co-authored more than 70 papers in international journals, books, and conference proceedings.



R. Castro-López received the "Licenciado en Física Electrónica" degree (M.S. degree on Electronic Physics) and the "Doctor en Ciencias Físicas" (Ph.D. degree) from the University of Seville, Spain, in 1998 and 2005 , respectively. Since 1998, he has been working at the Institute of Microelectronics of Seville (CSIC-IMSECNM) of the Spanish Microelectronics Center, where he now holds the position of Tenured Scientist. His research interests lie in the field of integrated circuits, especially design and computer-aided design for analog and mixed-signal circuits. He has participated in several national and international R\&D projects and coauthored more than 50 international scientific publications, including journals, conference papers, book chapters and the book Reuse-based Methodologies and tools in the Design of Analog and Mixed-Signal Integrated Circuits (Springer, 2006).

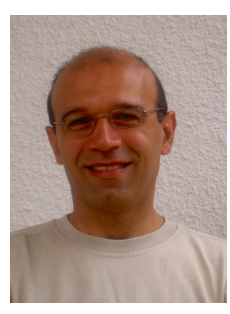

F.V. Fernández got the Physics-Electronics degree from the University of Seville, Spain, in 1988 and his Ph. D. degree in 1992. In 1993, he worked as a postdoctoral research fellow at Katholieke Universiteit Leuven (Belgium). From 1995 to 2009, he was an Associate Professor at the Dept. of Electronics and Electromagnetism of University of Seville, where he was promoted to full professor in 2009. He is also a researcher at IMSE-CNM (University of Seville and CSIC). Dr. Fernández was the Editor-in-Chief of Integration, the VLSI Journal (Elsevier) from 2005 to 2015. His research interests lie in the design and design methodologies of analog, mixed-signal and radiofrequency circuits. Dr. Fernández has authored or edited five books and has co-authored more than 150 papers in international journals and conferences. He has served as General Chair of three international conferences and regularly serves at the Program Committee of several international conferences. He has also participated as researcher or main researcher in numerous national and international R\&D projects. 